\title{
Inhibición de Colletotrichum gloeosporioides en cultivos de nañe en el Caribe colombiano usando aceites esenciales de Curcuma longa y Zingiber officinale
}

\author{
Inhibition of Colletotrichum Gloeosporioides in Yam Crops at the Colombian \\ Caribbean using Essential Oils of Curcuma Longa and Zingiber Officinale
}

\author{
Alexander Pérez Cordero ${ }^{1}$, Leonardo Chamorro Anaya ${ }^{2}$ y Jorge D. Mercado-Gómez ${ }^{3}$
}

\begin{abstract}
Resumen
Los aceites esenciales son compuestos orgánicos importantes en el sector farmacéutico debido a sus propiedades antimicrobianas, volviéndose una alternativa de solución para determinadas fitopatologías. En el presente estudio se evaluó in vitro la actividad inhibitoria de aceites esenciales de Curcuma longa y Zingiber officinale contra Colletotrichum sp. obtenidas a partir de cultivos de Dioscorea rotundata en Toluviejo (Sucre, Colombia). Concentraciones de 3,000; 5,000; 8,000 y 10,000 mg/L de extracciones de aceite (método de hidrodestilación asistida por microondas) fueron aplicados a cultivos in vitro del hongo. Además, se empleó un testigo absoluto y controles positivo y negativo. Los aceites fueron caracterizados por cromatografía de gases acoplado a espectrometría de masa. La actividad inhibitoria se realizó por medio de la prueba de siembra en superficie (porcentaje de índice de inhibición). Los mayores valores de índice antifúngico se observaron a 10,000 mg/L similar al obtenido con el control positivo por benomil. De la caracterización química de los aceites esenciales el metabolito secundario que se presentó con mayor rango fue el monoterpeno 3-Thujeno y el citral. Los aceites esenciales de $C$. longa y Z. officinale generaron actividad inhibitoria contra el crecimiento de Colletotrichum sp., y por lo tanto pueden ser empleados para reducir la antracnosis.
\end{abstract}

Palabras clave: citral, extraction, fungi, inhibition, angiosperms

\begin{abstract}
Essential oils are organic compounds during the last century have become important in the pharmaceutical sector because of its antimicrobial properties, being an alternative solution to certain plant diseases. In the present study, we evaluate in vitro the inhibitory activity of essential oils of Curcuma longa and Zingiber officinale against Colletotrichum sp. obtained from yam crops (Toluviejo, Sucre, Colombia). Concentrations 3,000; 5,000; 8,000 and 10,000 mg/L of each species of essential oils extractions were applied in vitro on the fungi (microwave assisted hydro-distillation method). In addition, we employed an absolute control, positive control (benomyl $1 \mathrm{~g} / \mathrm{L}$ ) and a negative control. The inhibitory activity was performed by seeding the test surface and expressed as percentage of inhibition index. We obtain than the highest percentages of antifungal index were observed at 10000 $\mathrm{mg} / \mathrm{L}$, similar to that obtained with the positive control. Of the chemical characterization of essential oils (gas chromatography coupled with mass spectrometry) the secondary metabolite that occurred more range was the monoterpene 3-Thujeno and citral. The essential oils of $C$. longa and Z. officinale show inhibitory activity against the growth of Colletotrichum sp., and therefore can be employed to reduce the use of chemical compounds to reduce the anthracnose.
\end{abstract}

Keywords: citral, extracción, hongo, inhibición, angiospermas

Recepción: 14-ene-2020

Aceptación: 12-dic-2020

\footnotetext{
${ }^{1}$ Doctor en Microbiología. Grupo Bioprospección Agropecuaria, Facultad de Ciencias Agropecuarias, Universidad de Sucre, Colombia.

${ }^{2}$ M.Sc. Grupo Bioprospección Agropecuaria, Universidad de Sucre, Colombia.

${ }^{3}$ Ph.D. Grupo Evolución y Sistemática Tropical, Departamento de Biología y Química. Universidad de Sucre, Colombia. Autor de correspondencia: jorge.mercado@unisucre.edu.co
} 


\section{Introducción}

En los trópicos se han identificado desde poblaciones de cazadores-recolectores diferentes especies de plantas que han sido utilizadas como sustento alimenticio [1]. Este proceso ha llevado a que muchas especies, hayan sido domesticadas por parte del hombre, de tal forma que se ha generado una dependencia entre el hombre y las plantas [2]. En este sentido, una especie vegetal de gran importancia nutricional que ha sido utilizada desde el Holoceno hasta el presente es el ñame [2]. Taxonómicamente las especies de este tubérculo corresponde al género Dioscorea, el cual pertenece a la familia de monocotiledóneas Dioscoreaceae [3] y está representado en el Caribe colombiano por diferentes variedades que no han sido clasificadas taxonómicamente [4].

En Colombia según reportes elaborados en 2010 se ha establecido que anualmente se producen alrededor 295.374 ton de ñame, lo cual ha llevado a incluir al territorio nacional dentro de los 12 países con mayor producción en las Américas. Este cultivo, es producido principalmente en departamentos como Córdoba, Bolívar, Atlántico y Sucre, donde es considerado de consumo básico en comunidades campesinas, y por lo tanto, de suma importancia socioeconómica para esta región [5]. Asimismo, es empleado en el abastecimiento de mercados locales y alimento para animales [6]. No obstante, y a pesar de su importancia en la economía local del Caribe colombiano, son poco los estudios que se han realizado para mejorar su calidad y producción, la cual se ha visto afectada en los últimos años por diferentes enfermedades producidas por hongos que han causado pérdidas de más del $90 \%$ en cultivos [12]. Bajo este contexto, el tubérculo del ñame se ha visto afectado por la incidencia principalmente de Colletotrichum gloeosporioides [(Penz.) Penz. y Sacc.] [7].

C. gloeosporioides es una especie de hongo cuya identidad taxonómica ha estado sujeta a variaciones debido a la dificultad en su identificación y similitudes morfológicas hacia otros taxones [8, 9]. Sin embargo, en términos generales este linaje ha sido establecido dentro de la familia Glomerellaceae y su distribución se encuentra en las regiones tropicales y subtropicales del mundo [10]. Este hongo es el causante de la enfermedad conocida como antracnosis [10], la cual reduce la eficiencia fotosintética de la planta hasta ocasionar disminución de la calidad de los tubérculos y una reducción de alrededor del $90 \%$ de la producción $[7,10]$. C. gloeosporioides también es causante de grandes pérdidas económicas en diferentes cultivos como frutales, cereales, pastos, vegetales, legumbres y cultivos perennes [11]. Además, ha sido reportado en cultivos de guanábana (Annona muricata L.) causando hasta el $90 \%$ de pérdidas en huertos no tecnificados [12]. De igual forma, se ha registrado en tomate de árbol [Cyphomandra betacea (Cav.) Sendt.], manzano (Malus domestica Borkh) y mora (Rubus glaucus Benth), dentro de los cuales ha provocado reducciones productivas superiores al $50 \%$ [13]. La antracnosis en al aguacate (Persea americana Mill.) ha afectado la calidad de los frutos y pérdidas cercanas al 20\% de la producción [14].

En este contexto, diferentes mecanismos han sido empleados para mitigar, controlar y exterminar las poblaciones de C. gloeosporioides [15]. Sin embargo, diferentes estudios han demostrado que los compuestos químicos utilizados en el control de este hongo como el Benomil producen problemas ambientales, en la salud de humanos [16], animales y en la diversidad microbiana [15]. En este sentido, sobre los últimos años se vienen implementando diferentes compuestos orgánicos biológicamente activos, extraídos de especies de plantas que presentan la capacidad de eliminar a microorganismos patógenos por sí mismas, debido al desarrollo de antibióticos [17]. Estos han demostrado su eficiencia al momento de mitigar este hongo $[18,19]$ y con esto reducir el impacto ambiental de las fumigaciones.

De los compuestos identificados como agentes químicos o en este caso antibióticos, se encuentran principalmente los aceites esenciales o también llamados compuestos volátiles [20]. Estos son considerados líquidos de consistencia aceitosa, aromáticos de origen vegetal (flores, los brotes, semillas, hojas, corteza, hierbas, madera, frutas y raíces), compuestos por sustancias lipofílicas, especialmente monoterpenos y sesquiterpenos, y 
sus derivados oxigenados (alcoholes, aldehídos, cetonas, fenoles, ácidos, ésteres y éteres) en distintas cantidades $[20,21]$.

A la fecha se han encontrado aceites esenciales en una gran variedad de plantas a las cuales se les ha probado cierta actividad antimicrobiana [22], incluyendo Zingiberaceae [23, 24]. Esta familia es un clado de monocotiledóneas perteneciente al orden Zingiberales, la cual cuenta con más de mil especies segregadas en 50 géneros y cuya distribución es pantropical [3]. En linajes al interior de esta familia diferentes estudios han demostrado su utilidad como agentes repelentes en mosquitos [23, 25], actividad antimicrobiana [26, 27] y anti-fúngica [24, 28, 29]. En este sentido, dos especies relevantes en términos de su importancia económica y etnobotánica se encuentran Curcuma longa L. y Zingiber officinale Roscoe. Según lo anterior, a las plantas de $C$. longa o como es conocida en la región del Caribe colombiano en su nombre vernáculo como cúrcuma se le atribuyen propiedades antioxidantes [30], hepatoprotectoras [31], anticancerígenas [32] y quimioprotectoras [33]; además se han hallado aceites esenciales en las raíces que corresponden a una mezcla de terpenos y turmerona como componentes mayoritarios [34]. Componentes que han sido registrados con agentes potenciales de actividad antimicrobiana [35]. Con respecto a Z. officinale o también denominado en la región caribeña en Colombia como Jengibre se ha estudiado que contiene hasta 3\% de un aceite esencial aromático, cuyos principales constituyentes son sesquiterpenoides, con zingibereno como el elemento principal; también su olor y sabor característicos son causados por una mezcla de zingerona, shogaoles y gingeroles a los cuales se ha reportado ciertas propiedades antibacterianas [26, 29].

En el departamento de Sucre se está implementando un programa para el aprovechamiento de recursos naturales según el conocimiento etnobotánico [5], para ser utilizados como potencial biológico, realizando estudios que permitan el descubrimiento de moléculas bioactivas novedosas, las cuales pueden ser halladas a partir de fuentes vegetales, como la Curcuma longa y Zingiber officinale, cuyo potencial biológico aunque ha sido establecido como fungicida $[36,37]$ aún se desconoce en cuanto a la inhibición de Colletotrichum gloeosporioides (Penz.) Penz. y Sacc en el cultivo de ñame. Según lo anterior, en el presente artículo se evalúa el potencial biológico de los aceites esenciales extraídos de las raíces de estas dos especies de monocotiledóneas sobre C. gloeosporioides, identificado en cultivos de Dioscorea rotundata Poir. desarrollados en el departamento de Sucre, Colombia. Para lo anterior, se llevó a cabo una caracterización química de los compuestos naturales, los cuales fueron probados sobre cepas del hongo obtenidas e identificadas a partir de D. rotundata.

\section{Materiales y métodos}

\subsection{Recolección del material vegetal, fúngico y verificación taxonómica}

Las muestras de Curcuma longa y Zingiber officinale fueron recolectadas en el municipio de Toluviejo (Sucre), en la zona rural de la Piche (Figura 1). El área de muestreo presenta una temperatura de $26^{\circ}$ y $30^{\circ} \mathrm{C}$, la precipitación anual es de $1.500 \mathrm{~mm}$ y la humedad relativa se encuentra entre $75 \%$ y $85 \%$. El régimen pluviométrico es bimodal, con valores máximos en los meses de septiembre a noviembre y mayo a junio [38]. La vegetación corresponde a bosque seco tropical, aunque actualmente solo existen pequeños parches de bosque aislados y fragmentados, de tal forma que paisaje corresponde a cultivos de orden comercial como Manihot esculenta (yuca), Carica papaya (papaya), entre otros.

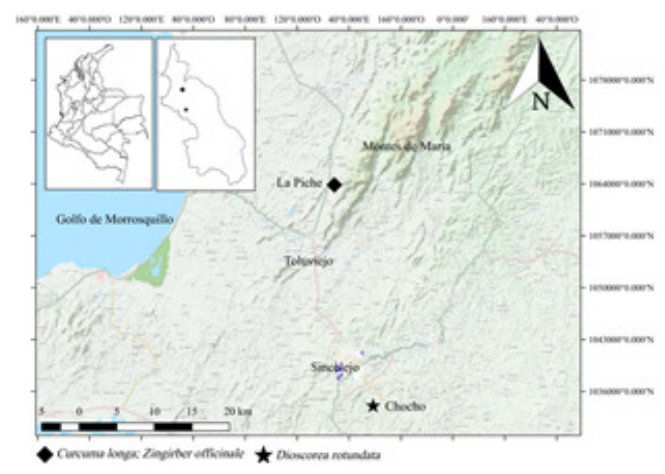

Figure 1. Localización de los sitios de muestreo en el departamento de Sucre (Colombia). 
Una vez recolectado el material, fue procesado según los parámetros convencionales del herbario en su secado y prensado; además su identificación taxonómica fue corroborada en el herbario de la Universidad de Sucre (HEUS) y almacenadas según la enumeración de Mercado-Gómez J.

Las cepas para los ensayos antifúngicos fueron aisladas a partir de hojas de ñame espino (nombre vernáculo) provenientes de cultivos en la vereda el Choncho $\left(-75^{\circ} 24^{\prime} 48.5^{\prime \prime}-9^{\circ} 30^{\prime} 46.11^{\prime \prime} \mathrm{N}\right)$ del municipio de Sincelejo (Figura 1). Una vez aislado el fitopatógeno según la metodología de Saldarriaga et al., (2008), las cepas de los hongos fueron enviadas a CorpoGen - Bogotá, Colombia para la extracción de DNA e identificación molecular.

\section{Extracción de aceites esenciales y análisis químico}

La obtención de los aceites esenciales se realizó por el método de hidrodestilación asistida por microondas (MWHD), empleando un equipo de hidrodestilación con capacidad para 2 L (balón de destilación). Se pesaron $300 \mathrm{~g}$ de material vegetal fresco (raíces), en buen estado; es decir, carente de evidencias de malformaciones o presencia de parásitos u otros. Este fue introducido en un balón de destilación con $800 \mathrm{~mL}$ de agua destilada. El tiempo de extracción fue de 1 hora para todas las muestras. Como fuente de radiación microondas se empleó un horno convencional, con un ciclo de irradiación de 60 minutos a una potencia de $70 \%$ $[39,40]$. Los aceites esenciales obtenidos, fueron secados con sulfato de sodio anhídrido y conservados en viales sellados a $4{ }^{\circ} \mathrm{C}$ protegidos de la luz, hasta su posterior utilización [41]. Posteriormente fue medido el rendimiento $(\% \mathrm{w} / \mathrm{w})$ del extracto, según el método propuesto por Díaz et al. [42] mediante la siguiente ecuación:

$$
\text { Rendimiento }(\%)=\left(\frac{W_{a}}{W_{b}}\right) \times 100
$$

Donde $W_{a}$ corresponde al peso del extracto seco, y $W_{b}$ al peso del material vegetal seco y molido.

Finalizada la extracción y medición del rendimiento, se procedió a determinar los componentes químicos de los aceites esenciales mediante la técnica instrumental de Cromatografía de Gases con detector selectivo de Masa (GC/MS), utilizando un equipo Agilent $6890 \mathrm{~N}$ acoplado a un detector selectivo de masa Agilent $5973 \mathrm{~N}$ y con el inyector en modo splitless. Los índices de Kóvats fueron determinados en una columna capilar no polar DB_1MS y utilizando como gas de arrastre el Helio. La programación del horno se dividió en cuatro pasos: inicialmente la temperatura fue de $50{ }^{\circ} \mathrm{C} \mathrm{y}$ se mantuvo allí por $0,33 \mathrm{~min}$, luego se incrementó la temperatura a razón de $5{ }^{\circ} \mathrm{C} / \mathrm{min}$ hasta $150{ }^{\circ} \mathrm{C}$ y se mantuvo allí por 4,34 min. Posteriormente, se incrementó la temperatura de $10^{\circ} \mathrm{C} / \mathrm{min}$ hasta 250 ${ }^{\circ} \mathrm{C}$ y se mantuvo por $3 \mathrm{~min}$ en la última. Por último, se incrementó la temperatura a razón de $15{ }^{\circ} \mathrm{C} / \mathrm{min}$ hasta $300{ }^{\circ} \mathrm{C}$ y se mantuvo por $4 \mathrm{~min}$. Este proceso tomo un tiempo total de $45 \mathrm{~min}$.

La identificación tentativa de los compuestos registrados se estableció según sus espectros de masas, usando la base de datos que presente una mayor probabilidad de coincidencia para las NIST02.L y NIST5a.L mayores a $90 \%$ o en su defecto usando la información de NIST98. L. Toda la información importante como tiempo de retención y porcentaje de área fue generada por el software MSD ChemStation.

\subsection{Inhibición del crecimiento micelial}

Para la prueba de inhibición del crecimiento micelial se siguió la metodología de siembra directa propuesta por Pérez et. al [43], con modificaciones. En esta prueba se utilizaron aislados de aproximadamente $7 \mathrm{~mm}$ de diámetro de área de crecimiento del fitopatógeno, los cuales se sembraron sobre la superficie del medio, papa destroza y agar enriquecido con cloranfenicol, rifampicina y ampicilina. Con el fin de establecer las posibles concentraciones de susceptibilidad del hongo ante los aceites, se elaboraron cuatro tratamientos de 3,000, 5,000, 8,000 y $10,000 \mathrm{mg} / \mathrm{L}$ de aceites de $C$. longa y Z. officinale, con el objeto que los aislados quedarán impregnados. De cada tratamiento, $60 \mu \mathrm{L}$ fueron adicionados a los aislados de $7 \mathrm{~mm}$ del fitopatógeno. Los tratamientos fueron comparados con el control positivo, los cuales presentaban benomil $1 \mathrm{~g} / \mathrm{L}$, bajo la dosis utilizada 
por los cultivadores de ñame en el departamento de Sucre; además, se empleó un testigo absoluto sin ningún tipo de tratamiento.

Los ensayos se incubaron de $28 \pm 2{ }^{\circ} \mathrm{C}$ por 8 días en intervalos de 12 horas de luz y 12 horas de oscuridad. La actividad antifúngica se evaluó midiendo el crecimiento radial de cada aislado con los diferentes tratamientos en el día octavo. El resultado se interpreta como porcentaje de índice antifúngico mediante la siguiente ecuación:

$$
\% I . A=\left[1-\frac{D_{a}}{D_{b}}\right] \times 100
$$

Donde $D_{a}$ corresponde al crecimiento de cada tratamiento y $D_{b}$ al crecimiento del testigo absoluto [44].

\subsection{Prueba múltiple de rango para el porcentaje de índice antifúngico (\% I.A)}

Para establecer si existían diferencias significativas entre las pruebas elaboradas a partir de los aceites y un testigo, se llevó a cabo un diseño completamente al azar con 3 réplicas por tratamiento. En este sentido, se determinaron tres factores experimentales: las concentraciones $(3,000,5,000$, 8,000 y $10,000 \mathrm{ppm}$ ), los bioproductos (aceites de C. longa y Z. officinale), cepas de hongos de Colletotrichum sp. y una variable respuesta que fue el porcentaje de índice antifúngico sobre el crecimiento micelial, para un total de 384 unidades experimentales. En todos los tratamientos, se adiciono una prueba positiva en contra del fitopatógeno a través del benomil.

Para contrastar la normalidad de los datos se realizó el test de Shapiro-Wilkse $(p=0,06016333)$; una vez verificada la normalidad de los datun un ANOVA con arreglo factorial simple con muestreo en las unidades experimentales se llevó a cabo. Finalmente, se realizó la prueba de Tukey para comparar la diferencia entre la media de los niveles de los factores y así establecer cuales factores presentaron las diferencias significativas. Todos los análisis estadísticos fueron elaborados en el programa PAST [45].

\section{Resultados}

\subsection{Recolección del material vegetal, fúngico e identificación taxonómica}

La verificación taxonómica de las muestras vegetales confirmó que de los ejemplares recolectados en campo corresponden a Curcuma longa L., Zingiber officinale Roscoe y Dioscorea rotundata Poir. En el caso de las cepas de hongo, los resultados del análisis molecular de las 16 cepas de objeto de estudio, mostraron un $100 \%$ de identidad de su longitud y similitud con secuencias de ITS almacenadas en banco genómicos (GeneBank) que corresponden a Colletotrichum gloeosporioides.

\subsection{Extracción de aceites esenciales y análisis químico}

Mediante la hidrodestilación se realizaron seis extracciones del aceite esencial a partir $300 \mathrm{~g}$ de cada una de las dos especies durante una hora, obteniéndose en promedio por cada extracción 0.50 $\mathrm{g}$ de un líquido amarillo pálido para los rizomas de C. longa, con un rendimiento de $0.25 \%$ (Tabla 1); y $0.85 \mathrm{~g}$ de un líquido color amarillo verdoso para los rizomas de $Z$. officinale, con un rendimiento de 0.43 $\%$ (Tabla 2).

Tabla 1. Resultados de las extracciones mediante hidrodestilación para los rizomas de C. longa y $Z$. officinale.

\begin{tabular}{ccccc}
\hline Extracciones & \multicolumn{2}{c}{ Masa de Aceite $(\mathrm{g})$} & \multicolumn{2}{c}{ Rendimiento (\%) } \\
\hline & C. longa & Z. oficcinale & C. longa & Z. oficcinale \\
1 & 0.50 & 0.86 & 0.25 & 0.43 \\
2 & 0.48 & 0.88 & 0.24 & 0.44 \\
3 & 0.52 & 0.86 & 0.26 & 0.43 \\
4 & 0.49 & 0.85 & 0.245 & 0.425 \\
5 & 0.51 & 0.85 & 0.255 & 0.425 \\
6 & 0.50 & 0.86 & 0.25 & 0.43 \\
\hline
\end{tabular}

Los resultados del análisis químico de los dos tipos de aceites muestran que hay una gran variabilidad de compuestos (Tabla 2 y 3 ). Para el caso de $C$. longa se presentan componentes mayoritarios al 3-Thujeno (25.57 \%) y Terpinoleno (19.09\%) (Tabla 2); mientras que en Z. officinale el citral $(15.40 \%)$ presenta la mayor concentración (Tabla 4). 
Tabla 2. Composición química del aceite esencial de Curcuma longa. Los compuestos marcados con asterisco presentan mayor cantidad en los aceites.

\begin{tabular}{lcc}
\hline Identificación & Tiempo de reacción & \% área \\
\hline Pineno & 7.140 & 5.13 \\
$\beta$-Pineno & 7.528 & 1.57 \\
3-Thujeno & $8.040^{*}$ & $25.57^{*}$ \\
Terpileno & 8.465 & 5.25 \\
Eucaliptol & 8.779 & 7.98 \\
trans- $\beta$-Ocimeno & 9.050 & 0.75 \\
$\gamma$-Terpineno & 9.364 & 3.25 \\
Terpinoleno & $10.374 *$ & $19.09^{*}$ \\
1,5,8-p-Mentatrieno & 10.710 & 0.08 \\
cis-p-Ment-2-en-1-ol & 10.901 & 0.12 \\
4-Metil Ciclohexeno & 11.925 & 0.21 \\
4-Carvomentenol & 12.327 & 1.12 \\
Sabinol & 12.890 & 0.35 \\
trans-Piperitol & 13,095 & 0.11 \\
1-(2-metoxi-fenil)-etanol & 13.805 & 0.79 \\
p-ment-1-en-3-ona & 14.032 & 0.13 \\
Linalool butirato & 14.434 & 0.32 \\
2-Undecanona & 15.341 & 0.29 \\
Etanona, 1-(2-tienil) & 15.905 & 0.92 \\
Metil cinnamato & 17.302 & 0.38 \\
Geranil acetato & 17.587 & 0.20 \\
3-Metil-5-pirazolidinona & 17.777 & 0.34 \\
Cariofileno & 18.897 & 0.33 \\
cis- $\beta$-Farneseno & 19.796 & 1.76 \\
$\alpha$-Farneseno & 21.011 & 0.98 \\
$\beta$-Sesquifelandreno & 21.442 & 0.43 \\
Nerolidol & 22.445 & 0.32 \\
$\alpha$-Bisabolol & 23.279 & 0.15 \\
cis- $\beta$-Santalol & 23.710 & 0.15 \\
\hline & 24.149 & 0.27 \\
Curlona & 25.985 & 7.12 \\
& & 3.07 \\
\hline
\end{tabular}

\subsection{Inhibición del crecimiento micelial}

Los aceites esenciales obtenidos de C. longa y $Z$. officinale, presentaron mayor actividad antifúngica a concentración de $10000 \mathrm{mg} / \mathrm{L}$ contra las cepas del hongo $C$. gloeosporioides con un índice antifúngico de $85 \%$, efecto similar al encontrado para el control positivo con benomil (\% I.A de $100 \%$ ). Las concentraciones de los aceites de 3,000 mg/L presentaron inhibición del $36 \% ; 5,000 \mathrm{mg} / \mathrm{L}$ con un $45 \%$ y $8,000 \mathrm{mg} / \mathrm{L}$ con un $60 \%$ contra $C$. gloeosporioides (Figura 2).

Tabla 3. Composición química del aceite esencial de Zingiber officinale. Los compuestos marcados con asterisco presentan mayor cantidad en los aceites.

\begin{tabular}{llc}
\hline Identificación & $\begin{array}{l}\text { Tiempo de } \\
\text { reacción }\end{array}$ & \% área \\
\hline 1R- $\alpha$-Pineno & 6.189 & 1.91 \\
Canfeno & 6.548 & 4.38 \\
$\beta$-Pineno & 7.491 & 1.50 \\
$\alpha$-Felandreno & 7.784 & 0.33 \\
$\gamma$-Terpineno & 8.567 & 7.65 \\
Linalool & 10.248 & 1.23 \\
Borneol & 11.991 & 1.88 \\
(+)- $\alpha$-Terpineol (p-ment-1-en-8-ol) & 12.642 & 2.39 \\
Citral & $15.261 *$ & $15.40 *$ \\
2,6-Dimetil-2,6-octadien-8-il acetato & 17.777 & 1.93 \\
Curcumeno & 20.433 & 4.21 \\
Biciclo [3.1.1]hept-2-eno, 2,6-dimetil- & 20.959 & 5.99 \\
6-(4-metil-3-pentenil) & & \\
1,3-Ciclohexadieno, 5-(1,5-dimetil-4- & 21,091 & 4,94 \\
hexenil)-2-metil-, [S- (R*, S*)]- & & \\
$\alpha$-Farneseno & 21.384 & 5.95 \\
$\beta$-Sesquifelandreno & 21.823 & 4.53 \\
Eremofileno (7CI) & 22.598 & 1.41 \\
Dehidrolinalool & 24.376 & 1.36 \\
$\beta$-Eudesmol & 25.473 & 1.13 \\
$\alpha$-Cedreno & 26.622 & 1.91 \\
\hline & & \\
\hline
\end{tabular}

\subsection{Prueba múltiple de rango para el porcentaje de índice antifúngico (\% I.A)}

Se encontraron diferencias significativas entre las concentraciones de los dos aceites evaluados contra C. gloeosporioides, puesto que el p-valor está por debajo de 0.05 con un nivel de confianza del $95 \%$. Las pruebas estadísticas (Tukey) indican diferencias significativas con respecto a la concentración de los aceiteses enciales. Los mayores índices de inhibición (85\%) en ambos aceites fueron encontrados cuando se utilizaron concentraciones de $10,000 \mathrm{mg} / \mathrm{L}$ con respecto a los menores \% I.A, quienes fueron observadas a de 3,000 y $5,000 \mathrm{mg} / \mathrm{L}$, respectivamente. El \% I.A del volumen $10000 \mathrm{mg} / \mathrm{L}$ fue muy similar al control positivo con benomil. 


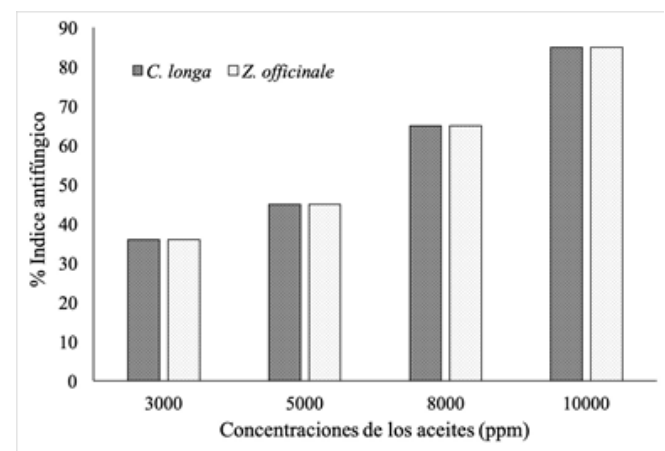

Figure 2. Concentraciones de los aceites extraídos de C. longa y Z. officinale y su relación con la inhibición a través del \% del índice antifúngico.

\section{Discusión}

Los aceites esenciales de $C$. longa y $Z$. officinale reportaron un rendimiento promedio de $0.25 \%$ y $0.43 \%$ respectivamente, esto en relación a los mililitros obtenidos por gramo de muestra fresca. Estos resultados de rendimiento muestran un porcentaje de producción relativamente bajo en el proceso de extracción de los aceites al compararlos con otros estudios, como el realizado por Mesa, et al. [34], quienes hallaron niveles superiores en su investigación. Lo anterior puede ser el producto de las diferentes condiciones tanto del material vegetal como de las condiciones ambientales en que se encuentre el cultivo. Barbosa, et al. [46] reporta que al someter la planta a un proceso de secado se reduce entre un $12 \%$ y un $17 \%$ del contenido de aceite en comparación con el contenido del mismo en la planta fresca, debido a la volatilización de una parte del aceite en el proceso. Mejía, et al. [47] y Delgado, et al. [48], reportan que el contenido de humedad del material vegetal al momento de la extracción es un factor importante en el rendimiento de los aceites esenciales ya que afecta la concentración de los mismos.

Del mismo modo, Fornari, et al. [20], también indicaron que los factores ambientales, como las propiedades del suelo y el estrés por el agua o la temperatura en la zona cultivada tienen influencia en la productividad de los aceites por parte de las especies, pero tienen poco efecto en la composición del aceite esencial. Sin embargo, estos resultados de rendimiento son satisfactorios debido que sus valores están por encima de $0.1 \%$ que es dato límite mínimo de rendimiento según Joulain [49]; en otras palabras, aunque los valores son inferiores a los reportados en otros estudios, es posible proponer su explotación desde el punto de vista industrial.

Los aceites esenciales obtenidos de $C$. longa y $Z$. officinale, presentaron mayor actividad antifúngica a concentración de 10,000 mg/L contra las cepas del hongo $C$. gloesporioides con un índice antifúngico de $85 \%$, efecto similar al encontrado para el control positivo con benomil (\% I.A de $100 \%$ ). Se puede inferir que la concentración mínima inhibitoria se encuentre en este valor $(10,000 \mathrm{mg} / \mathrm{L})$. No obstante, algunos investigadores han observado, que los aceites de $C$. longa pueden tener un efecto positivo a concentraciones más bajas $[50,51]$, por ejemplo, se pudo determinar que $5,000 \mathrm{mg} / \mathrm{L}$ genera una actividad del $70 \%$. Empero, al igual que en presente estudio, Sitara, et al. [52] al comparar la efectividad del aceite esencial de $C$. longa con fungicidas comerciales contra Aspergillus flavus, observaron una total de inhibición del crecimiento del hongo a concentraciones de aceite de 10,000 $\mathrm{mg} / \mathrm{L}$.

En la composición química del aceite esencial de cúrcuma, este mostró la presencia de monoterpenos y sesquiterpenos. Chen, et al. [53] establecieron que los extractos de $C$. longa generan efectos inhibitorios contra el hongo Fusarium graminearum, generando la rotura de la membrana celular fúngica y la inhibición de la síntesis de ergosterol. De igual forma, Avano et al. [54] reportaron actividad antifúngica sobre Fusarium verticillioides, en el cual la producción de ergosterol disminuyo significativamente, indicando un efecto del aceite en la biomasa del hongo; en otras palabras, los aceites presentaron propiedades antioxidantes. Asimismo, $\mathrm{Hu}$, et al. [55] también establecieron más evidencias de la actividad antifúngica de $C$. longa, determinando un efecto relacionado a la disrupción del sistema de endomembrana celular.

En el caso de Z. officinale son varios los estudios que han demostrado su eficiencia en contra de diferentes fitopatógenos [56, 57]. AniediAbasi Akpan, et al. [58] encontraron su eficacia al momento de reducir la biomasa micelial de Lasiodiplodia theobromae en especies de $D$. 
rotundata. Del mismo modo, Gwa and Nwankiti [59] evaluaron aceites esenciales de diferentes especies, incluyendo $Z$. officinale en cepas de Colletotrichum sp. extraídas de D. rotundata. Gwa and Nwankiti [59] también pudieron establecer que el aceite de $Z$. officinale es el más eficiente en contra del crecimiento de la biomasa del hongo; no obstante, dado que estos autores solo llegaron a nivel de género en la identificación del hongo, se desconoce sobre cual especie los resultados de eficacia antifúngica son tan altos. De esta forma en el presente estudio, dado que fue posible llegar a la identificación taxonómica a través de herramientas molecular de la especie C. gloesporioides, los resultados aquí obtenidos muestran que los aceites de Z. officinale son una alternativa de fácil acceso y uso por parte de comunidades rurales para mitigar la expansión de la antracnosis.

\section{Conclusiones}

Los resultados obtenidos en esta investigación muestran la importancia que tienen los aceites esenciales en la búsqueda de nuevas alternativas para el control de fitopatógenos que afectan los cultivos de interés agrícola. En este sentido, es posible asumir que los aceites esenciales de C. longa y $Z$. officinale presentaron actividad inhibitoria contra C. gloesporioides. Por lo tanto, se abre un área de investigación que ayude a buscar alternativas biológicas a contrarrestar los efectos de estos fitopatógenos en cultivos de $D$. rotundata, convirtiéndose este estudio in vitro en el primer reporte a nivel nacional como una alternativa promisoria para el manejo de la antracnosis causada por especies de $C$. gloesporioides en cultivo de $D$. rotundata.

Estos aceites esenciales mostraron como compuestos mayoritarios 3-Thujeno y citral respectivamente; no obstante, es necesario realizar pruebas más profundas, extrayendo estos compuestos y detallando su efectividad sobre el hongo. Ya que en el caso del citral se ha demostrado que tiene gran capacidad para inhibir el crecimiento microbial [60]. Del mismo modo, con base en los resultados obtenidos de antagonismos es posible sugerir que estos aceites pueden ser una alternativa biológica amigable con el ecosistema para el control de la antracnosis en los cultivos de D. rotundata en el departamento de Sucre, Colombia.

\section{agradecimientos}

Los autores agradecen a la Universidad de Sucre por toda su colaboración en la ejecución de la investigación.

\section{Referencias}

[1] F. J. Aceituno and N. Loaiza, "Early and Middle Holocene evidence for use of plants and cultivation in the the Middle Cauca River Basin, Cordillera Central (Colombia)," Quaternary Science Reviews, vol. 86, pp. 49-62, 2014.

[2] D. R. Piperno, "The Origins of Plant Cultivation and Domestication in the New World Tropics Patterns, Process, and New Developments," Current Anthropology, vol. 52, pp. S453-S470, 2011.

[3] M. G. Simpson, Plant systematics Burlington: Elsevier Academic Press, 2010.

[4] R. Bernal, G. Galeano, A. Rodríguez, S. H, and G. M. (2016). Nombres Comunes de las Plantas de Colombia. http://www.biovirtu al.unal.edu.co/nombrescomunes/. Available: http://www.biovirtual.unal.edu.co/nombresco munes/

[5] E. Barrios-Paternina and J. Mercado-Gómez, "Plantas útiles del corregimiento Santa Inés y vereda San Felipe-San Marcos (Sucre, Colombia)," Ciencia en desarrollo, vol. 5, pp. 131-144, 2014.

[6] Y. Reina, El Cultivo del ñame en el Caribe Colombiano vol. 3: Banco de la República. Centro de Estudios Económicos Regionales (CEER)-Cartagena. Documento de trabajo sobre economía regional, 2012.

[7] Y. Pinzón, S. Bustamante, and G. Buitrago, "Diagnóstico molecular diferencial de Colletotrichum gloeosporioides y Fusarium oxysporum en ñame (Dioscorea sp.)," Revista 
Colombiana de Biotecnología vol. 15, pp. 5260, 2013.

[8] B. S. Weir, P. R. Johnston, and U. Damm, "The Colletotrichum gloeosporioides species complex," Studies in Mycology, vol. 73, pp. 115-180, 2012.

[9] P. S. Gunnell and W. D. Gubler, "Taxonomy and morphology of Colletotrichum species pathogenic to Strawberry," Mycologia, vol. 84, pp. 157-165, 1992.

[10] Y. Méndez, J. Palencia, K. Hernández, E. Hernández, and B. J., "Reacción de genotipos de ñame (Dioscorea spp.) a la antracnosis (Colletotrichum gloeosporioides)," Tema Agrarios, vol. 18, pp. 34-40, 2013.

[11] L. Afanador-Kafuri, D. Minz, M. Maymon, and S. Freeman, "Characterization of Colletotrichum Isolates from Tamarillo, Passiflora, and Mango in Colombia and Identification of a unique species from the genus," Phytopathology, vol. 93, pp. 579-587, 2003.

[12] E. Álvarez, A. Ospina, J. Mejía, and G. Llano, "Caracterización morfológica, patogénica y genética del agente causal de la antracnosis (Colletotrichum gloesporioides) en guanábana (Annona muricata) en el Valle del Cauca," Fitopatología Colombiana vol. 28, pp. 1-8, 2004.

[13] A. Saldarriaga, J. Castaño, and R. Arango, "Caracterización del agente causante de la antracnosis en tomate de árbol, manzano y mora.," Revista Colombiana de Ciencias Exactas Físicas y Naturales, vol. 32, pp. 145156, 2008.

[14] S. Rodríguez, M. González, and N. Mayek, "La Infección de Colletotrichum gloeosporioides (Penz.) Penz. y Sacc. en Aguacatero (Persea americana Mill.): Aspectos Bioquímicos y Genéticos. Revista Mexicana de Fitopatología, vol. 27, pp. 53-63, 2008.

[15] S. Cordeau, M. Triolet, S. Wayman, C. Steinberg, and J.-P. Guillemin, "Bioherbicides:
Dead in the water? A review of the existing products for integrated weed management,' Crop Protection, vol. 87, pp. 44-49, 2016.

[16] J. Zhou, K. Xiong, Y. Yang, X. Ye, J. Liu, and F. Li, "Deleterious effects of benomyl and carbendazim on human placental trophoblast cells," Reproductive Toxicology, vol. 51, pp. 64-71, 2015.

[17] D. J. Daferera, B. N. Ziogas, and M. G. Polissiou, "The effectiveness of plant essential oils on the growth of Botrytis cinerea, Fusarium sp. and Clavibacter michiganensis subsp. michiganensis," Crop Protection, vol. 22, pp. 39-44, 2003.

[18] N. D. Anaruma, F. L. Schmidt, M. C. T. Duarte, G. M. Figueira, C. Delarmelina, E. A. Benato, et al., "Control of Colletotrichum gloeosporioides (penz.) Sacc. In yellow passion fruit using Cymbopogon citratus essential oils," Brazilian Journal of Microbiology, vol. 41, pp. 66-73, 2010.

[19] A. B. Perumal, P. S. Sellamuthu, R. B. Nambiar, and E. R. Sadiku, "Antifungal activity of five different essential oils in vapour phase for the control of Colletotrichum gloeosporioides and Lasiodiplodia theobromae in vitro and on mango," International Journal of Food Science \& Technology, vol. 51, pp. 411-418, 2016.

[20] T. Fornari, G. Vicente, E. Vázquez, M. R. García-Risco, and G. Reglero, "Isolation of essential oil from different plants and herbs by supercritical fluid extraction," Journal of Chromatography A, vol. 1250, pp. 34-48, 2012.

[21] C. Coy Barreraa and D. Gómez, "Caracterización y variabilidad química de dos aceites esenciales de Croton funckianus (Euphorbiaceae)," Revista Ciencia en desarrollo vol. 6, pp. 155-160, 2015.

[22] K. Husnu Can Baser and G. Buchbauer, Handbook of Essential Oils: Science, Technology, and Applications, Second Edition ed.: CRS Press. Taylor \& Francis group, 2016. 
[23] T. Pushpanathan, A. Jebanesan, and M. Govindarajan, "The essential oil of Zingiber officinalis Linn (Zingiberaceae) as a mosquito larvicidal and repellent agent against the filarial vector Culex quinquefasciatus Say (Diptera: Culicidae)," Parasitology Research, vol. 102, pp. 1289-1291, 2008.

[24] D. Suthisut, P. G. Fields, and A. Chandrapatya, "Fumigant toxicity of essential oils from three Thai plants (Zingiberaceae) and their major compounds against Sitophilus zeamais, Tribolium castaneum and two parasitoids," Journal of Stored Products Research, vol. 47, pp. 222-230, 2011.

[25] L. S. Nerio, J. Olivero-Verbel, and E. Stashenko, "Repellent activity of essential oils: A review," Bioresource Technology, vol. 101, pp. 372-378, 1// 2010.

[26] K. Norajit, N. Laohakunjit, and O. Kerdchoechuen, "Antibacterial Effect of Five Zingiberaceae Essential Oils," Molecules, vol. 12, p. 2047, 2007.

[27] K. Cimanga, K. Kambu, L. Tona, S. Apers, T. De Bruyne, N. Hermans, et al., "Correlation between chemical composition and antibacterial activity of essential oils of some aromatic medicinal plants growing in the Democratic Republic of Congo," Journal of Ethnopharmacology, vol. 79, pp. 213-220, 2002.

[28] I. b. Jantan, M. S. M. Yassin, C. B. Chin, L. L. Chen, and N. L. Sim, "Antifungal Activity of the Essential Oils of Nine Zingiberaceae Species," Pharmaceutical Biology, vol. 41, pp. 392-397, 2003.

[29] G. Sacchetti, S. Maietti, M. Muzzoli, M. Scaglianti, S. Manfredini, M. Radice, et al., "Comparative evaluation of 11 essential oils of different origin as functional antioxidants, antiradicals and antimicrobials in foods," Food Chemistry, vol. 91, pp. 621-632, 2005.

[30] M. Cousins, J. Adelberg, F. Chen, and J. Rieck, "Antioxidant capacity of fresh and dried rhizomes from four clones of turmeric
(Curcuma longa L.) grown in vitro," Industrial Crops and Products, vol. 25, pp. 129-135, 2007.

[31] M. Sengupta, G. D. Sharma, and B. Chakraborty, "Hepatoprotective and immunomodulatory properties of aqueous extract of Curcuma longa in carbon tetra chloride intoxicated Swiss albino mice," Asian Pacific Journal of Tropical Biomedicine, vol. 1, pp. 193-199, 2011.

[32] M. López-Lázaro, "Anticancer and carcinogenic properties of curcumin: Considerations for its clinical development as a cancer chemopreventive and chemotherapeutic agent," Molecular Nutrition \& Food Research, vol. 52, pp. S103-S127, 2008.

[33] P. Anand, S. G. Thomas, A. B. Kunnumakkara, C. Sundaram, K. B. Harikumar, B. Sung, et al., "Biological activities of curcumin and its analogues (Congeners) made by man and Mother Nature," Biochemical Pharmacology, vol. 76, pp. 1590-1611, 2008.

[34] M. Mesa, M. Ramírez, C. Aguilera, A. Ramírez, and A. Gil, "Efectos farmacológicos y nutricionales de los extractos de Curcuma longa L. y de los cucuminoides," Ars Pharmaceutica, vol. 41, pp. 307-321, 2000.

[35] G. Asghari, A. Mostajeran, and M. Shebli, "Curcuminoid and essential oil components of turmeric at different stages of growth cultivated in Iran," Research in Pharmaceutical Sciences, vol. 4, pp. 55-61, 2009.

[36] G. Singh, O. P. Singh, and S. Maurya, "Chemical and biocidal investigations on essential oils of some Indian Curcuma species," Progress in Crystal Growth and Characterization of Materials, vol. 45, pp. 75-81, 2002.

[37] C. Darshana, R. Praveena, S. Ankegowda, and C. N. Biju, "Morphological variability, mycelial compatibility and fungicidal sensitivity of Colletotrichum gloeosporioides causing leaf spot of ginger (Zingiber officinale Rosc.)," 
Journal of Spices and Aromatic Crops, vol. 23, pp. 211-223, 2014.

[38] Promontes, Programa de Desarrollo y Paz de los Montes de María. Cartagena: Promontes, Corporación Territorios, Universidad de Cartagena, 2003.

[39] M. Torrenegra, C. Granados, M. Osorio, and G. León, "Method comparison of hydrodistillation microwave radiation-assisted (MWHD) front hydrodistillation (HD) in the extraction of essential oil of Minthostachys mollis," Inf. Tecnol., vol. 26, pp. 117-122, 2015.

[40] M. A. Ferhat, B. Y. Meklati, J. Smadja, and F. Chemat, "An improved microwave Clevenger apparatus for distillation of essential oils from orange peel," Journal of Chromatography A, vol. 1112, pp. 121-126, 2006.

[41] H. Rodríguez, P. Giraldo, and M. P., "Determinación del quimiotipo de la fracción volátil del aceite esencial de hojas de albahaca de variedad ocimum, por cromatografía de gases acoplada a masas (GC-MS).” Rev. Tumbaga vol. 6, pp. 53-62, 2011.

[42] P. Díaz, A. Cabrera, D. Alem, P. Larrañaga, F. Ferreira, and M. Dalla, "Antifungal activity of medicinal plant extracts against phytopathogenic fungus Alternaria spp.," Chilean Journal of Agricultural Research, vol. 71, pp. 231-239, 2011.

[43] A. Pérez, J. Rojas, L. Chamorro, and K. Pérez, "Evaluación in vitro de actividad inhibitoria de extractos vegetales sobre cepas de hongos del género Colletotrichum sp.," Acta Agronómica, vol. 60, pp. 158-164, 2011.

[44] Z. Guo, R. Xing, S. Liu, Z. Zhong, X. Ji, L. Wang, et al., "The influence of molecular weight of quaternized chitosan on antifungal activity," Carbohydrate Polymers, vol. 71, pp. 694-697, 2008.

[45] O. Hammer, D. A. T. Harper, and P. D. Ryan, "PAST: Paleontological statistics software package for education and data analysis," Palaeontologia Electronica, vol. 4, p. 9, 2001.
[46] F. d. F. Barbosa, L. C. A. Barbosa, E. C. Melo, F. M. Botelho, and R. H. S. Santos, "Influencia da temperatura do ar de secagem sobre o teor e a composicao química do óleo essencial de Lippia alba (Mill) N. E. Brown," Química Nova, vol. 29, pp. 1221-1225, 2006.

[47] O. Mejía, J. Marcial, M. Sánchez, C. Bonilla, and P. Vanegas, "Efecto de la altura y frecuencia de corte y secado en el rendimiento y calidad del aceite esencial de pronto alivio," Scientia tech, vol. 23, pp. 253-255, 2007.

[48] J. Delgado, M. Sánchez, and C. Bonilla, "Efecto del secado y la edad de las plantas en la composición de los aceites esenciales de Lippia alba (Mill.) N.E.Br. ex Britton \& P. Wilson y Lippia origanoides Kunth,” Acta Agronómica, vol. 65, pp. 170-175, 2015.

[49] D. Joulain, "Investigating New Essential Oils: Rationale, results and limitations," Perfumer \& Flavorist, vol. 21, pp. 1-10, 1996.

[50] A. K. Mishra and N. K. Dubey, "Evaluation of some essential oils for their toxicity against fungi causing deterioration of stored food commodities," Applied and Environmental Microbiology, vol. 60, pp. 1101-1105, 1994.

[51] R. K. Sharma, B. P. Misra, T. C. Sarma, A. K. Bordoloi, M. G. Pathak, and P. A. Leclercq, "Essential Oils of Curcuma longa L. from Bhutan," Journal of Essential Oil Research, vol. 9, pp. 589-592, 1997.

[52] U. Sitara, I. Niaz, J. Naseem, and N. Sultana, "Antifungal effect of essential oils on in vitro growth of pathogenic fungi," Pakistan Journal of Botany, vol. 40, pp. 409-414, 2008

[53] C. Chen, L. Long, F. Zhang, Q. Chen, C. Chen, $\mathrm{X}$. Yu, et al., "Antifungal activity, main active components and mechanism of Curcuma longa extract against Fusarium graminearum," PLOS ONE, vol. 13, p. e0194284, 2018.

[54] G. B. Avano, F. D. Ferreira, N. S. Bomfim, P. A. d. S. R. d. Santos, R. M. Peralta, T. Brugnari, et al., "Curcuma longa L. essential 
oil composition, antioxidant effect, and effect on Fusarium verticillioides and fumonisin production," Food Control, vol. 73, pp. 806813, 2017/03/01/ 2017.

[55] Y. Hu, J. Zhang, W. Kong, G. Zhao, and M. Yang, "Mechanisms of antifungal and anti-aflatoxigenic properties of essential oil derived from turmeric (Curcuma longa L.) on Aspergillus flavus," Food Chemistry, vol. 220, pp. 1-8, 2017/04/01/ 2017.

[56] I. López, M. Balcázar, J. Mendoza, A. Ortiz, M. Melo, R. Parrales, et al., "Antimicrobial Activity of Essential Oil of Zingiber officinale Roscoe ( Zingiberaceae ) Etna.," American Journal of Plant Sciences, vol. 8, 2017.

[57] O. Ayodele, F. Akinyosoye, D. Arotupin, O. Owoyemi, and A. Oyindamola, vPhytochemical Screening and Antifungal Activities of Zingiber officinale (Roscoe) on Mycotoxigenic Fungi Associated with the Deterioration of Pennisetum glaucum Grains," Journal of Advances in Microbiology, vol. 13 pp. 1-11, 2018.
[58] M. Aniedi-Abasi Akpan, O. Garuba, U. Etim Johnson, A. Anderson Chidi, and U. Samuel Effiong, "Potency of Plant-based Fungicides against a Rot Pathogen of White Yam (Dioscorea rotundata Poir)," Journal of Advances in Microbiology, vol. 5 pp. 1-9, 2017.

[59] V. Gwa and A. Nwankiti, "Efficacy of Some Plant Extracts in In Vitro Control of Colletotrichum Species, Causal Agent of Yam (Dioscorea rotundata Poir) Tuber Rot," Pelagia Research Library, vol. 7, pp. 8-16, 2017.

[60] R. Garcia, E. S. S. Alves, M. P. Santos, G. M. F. V. Aquije, A. A. R. Fernandes, R. B. d. Santos, et al., "Antimicrobial activity and potential use of monoterpenes as tropical fruits preservatives," Brazilian Journal of Microbiology, vol. 39, pp. 163-168, 2008. 